\title{
A cross-sectional study: medication safety among cancer in-patients in tertiary care hospitals in KPK, Pakistan
}

\author{
Marium Azim* (D), Ahmad Khan², Tahir Mehmood Khan ${ }^{3}$ and Mohammad Kamran
}

\begin{abstract}
Background: Medication safety in cancer patients receiving complex medication regimens is an important problem in various settings. Medication related events, interceptions and interventions are not well described in this area. We intended to study incidence, types, settings and stages involved, root cause analysis, medication classes involved and the level of harm cause by medication errors in two hospitals providing oncology services comparatively. The severity of incidents and interventions are studied.
\end{abstract}

Methods: It was a prospective cross sectional study among cancer in-patients of two tertiary care hospitals of KPK. Scale by NCC-MERP was used for evaluation of all medication related incidents. The data obtained was analyzed by IBM SPSS statistics 22 with 95\% confidence interval and used the same for other descriptive statistics.

Results: All medication orders were reviewed at both sites (Computerized Prescription Order Entry and HWP systems). Potential ADEs incidence was found high at site 2 (97.5\%) while medication errors without harm was high at site 1 (97.5\%). Most events occur at prescribing level 87.6 and $81.7 \%$ at both sites 1 and 2. Types highly reported involved improper dose 31.4 and 15.5\%, monitoring error 14.6 and $15.2 \%$ at site 1 and 2 . Medications involved in these incidents were antibiotics 44 and $12.7 \%$, antiemetic 7.5 and $15.8 \%$ and antineoplastic 2.9 and $9.4 \%$ at site 1 and 2. Severity of 3.6 and $36.5 \%$ incidents had potential to cause harm at site 1 and 2 . Root causes were human factors 62.6 and 72.3\%, drug selection 33.6 and 38.8\%, and dose selection 39.6 and $15.3 \%$ at sites 1 and 2 . Contributing factors including staff training 33.6 and $24.3 \%$, system for covering patient care 14.9 and $36.6 \%$, communication system 2.4 and 20.3\%, interruptions 9.7 and $7.3 \%$ and others 78.8 and $68.6 \%$ were highly reported. Preventability of medication errors was $99 \%$ at both sites. Intervention was taken in $90.5 \%$ events at site 1 (CPOE system) while the incidence lowest at site 2 (HWP system).

Conclusion: Medication related events are high among cancer in-patients at the site lacking updated electronic system for medication prescribing. Proper training about medication safety, reporting and interventions are required.

Keywords: Medication safety, Medication related events, Interventions, Root cause analysis, Contributing factors, Chemotherapy, Potential ADEs

\footnotetext{
* Correspondence: marium@bs.qau.edu.pk

${ }^{1}$ Quaid-I-Azam University, QAU Islamabad, Islamabad, Pakistan

Full list of author information is available at the end of the article
}

(c) The Author(s). 2019 Open Access This article is distributed under the terms of the Creative Commons Attribution 4.0 International License (http://creativecommons.org/licenses/by/4.0/), which permits unrestricted use, distribution, and reproduction in any medium, provided you give appropriate credit to the original author(s) and the source, provide a link to the Creative Commons license, and indicate if changes were made. The Creative Commons Public Domain Dedication waiver (http://creativecommons.org/publicdomain/zero/1.0/) applies to the data made available in this article, unless otherwise stated. 


\section{Background}

According to the Latin phrase "Premium non nocere" or "first, do no harm" is one of the main part of the oath the physicians take during practicing medicine. It is because of the complex and human nature of medicine management [1]. But one of the serious challenges to modern health care system is the occurrence of medication errors (MEs). MEs may cause patient harm, increased healthcare cost and ineffective use of health care resources [1-4]. These may also result in increased inpatient hospital days and risk of morbidity and mortality [5]. Patient safety improvement [5] became a global concern for all including healthcare professionals (HCPs), policy makers and the public [6]. The medication use process is highly prone to errors [5]. MEs among prescriptions and intravenous administration of drugs to the patients were highly reported [7-9]. The severity ranged from minor harm to life threatening incidents [1].

Attitudes like hiding errors or punitive approach causes difficulty in creating safe culture and patient safety among HCPs [1]. A change in culture approach greatly reduced error incidence from 11.4 to $7.3 \%$ [1]. 59\% errors had ability to cause harm, $25 \%$ actually caused harm while $30 \%$ were harmless during an academic study. The highest rate was reported in intensive care patients (21.1 per 100 admissions) during ordering (96\%) [6]. In 16-24\% of events MEs occurred either in cluster of sequence or in the form of groups. Highest rate was reported during ordering (32\%) and administration (39\%) [10].

Cancer patients are more prone to medication errors like prescription errors with over dosage. Anti-neoplastic medicines have several toxic effects at their therapeutic doses as well because of the narrow therapeutic index, complex regimes and the vulnerability of cancer patients to potential harms [2, 11-13]. All these factors make anti-neoplastic drugs the second most common cause of death. This fact makes it important to well recognize the predictors involved in medication errors [12]. Studies showed $>5 \%$ of chemotherapy orders contained at least one $\mathrm{ME}[12,13],>50 \%$ of administration errors [14], >40\% omission errors, $>20 \%$ errors due to brand names and due to abbreviations used for chemotherapy and pre-medications [11].

In this study we tried to evaluate the incidence of medication errors among cancer inpatients along with humanistic and system related causes and contributing factors. The medication errors were evaluated according to the scale established by National Coordinating Center for Medication Errors Reporting and Preventions (NCC-MERP).

\section{Methods}

\section{Study design}

The study was approved by the scientific committee of the institute and both hospital sites. The study design was cross-sectional and extended over the period of nine months at both sites. The medical records were directly observed by the principal investigator for all in-patients receiving chemotherapy.

\section{Study sites}

Both study sites were non-government health institutes. The pharmacy services at both institutes differed on the basis of system operation. Site 1 used highly upgraded software with computer order entry system (CPOE)/ computer decisions supportsystem (CDSS). While at Site 2 all medication orders were handwritten by the oncologists. The medication orders were forwarded by nurses through software to in-patient pharmacies while dispensed by pharmacists.

\section{Data collection tool}

Medication orders were reviewed after prescription by the principle investigator. Data collection tool was designed by the criteria of NCC-MERP. While medication orders were reviewed with several approved medical guidelines and literature such as Medscape, British National Formulary (BNF), National Comprehensive Cancer Network (NCCN) guidelines and other drug indices which were followed at the settings., More than one type, stage, causes and contributing factors were included for a medication order where needed. Where a number of causes and contributing factors lead to the occurrence of incident, these were reported separately.

\section{Eligibility criteria}

Medication orders of in-patients (both adults and pediatrics) with breast, ovarian, prostrate, blood and gastric cancer receiving chemotherapy and/or supportive care medications were reviewed. Patients receiving radiotherapy or anti-neoplastic drugs for indications other than cancer were excluded. Patients visiting multiple times for chemotherapy were included again.

\section{Data analysis}

We calculated the rate of incidents per 1000 medication orders. Mean with $95 \%$ confidence interval was obtained for the types, causes and contributing factors of incidents along with rate and a two-tailed significance via IBM SPSS statistics 22. Further descriptive analysis for the stages and medication classes involved in medication related events.

\section{Results}

In this study, 75 charts (in CPOE system) with 2925 medication orders at site 1 and 76 charts (in HWP system) with 1657 medication orders at site 2 were reviewed. A total of 1195 incidents were found during 
review of all medication orders $(n=4852)$. A rate of 0.25 incidents per 1000 orders occurred.

\section{Types of incidents at both sites}

At site 1 (CPOE system) approximately 185 incidents (31.4\%, CI $0.28-0.35, P=0.000)$ showed improper dose, 133 incidents $(22.6 \%$, CI $0.19-0.26, P=0.000)$ of potential problems with the lack of treatment effectiveness, 116 incidents $(19.7 \%, \mathrm{CI} 0.16-0.23, \mathrm{P}=0.000)$ of wrong drug, 86 incidents $(14.6 \%, C I 0.12-0.17, P=0.000)$ of monitoring errors including contraindications and drug interactions, 69 incidents $(11.7 \%, \mathrm{CI} 0.09-0.14, P=0.000)$ of wrong time or schedule of drug administration and 229 other types of incidents $(38.9 \%$, CI $0.35-0.43, P=$ 0.000 ) such as not highlighting administration considerations in 33 incidents (5.6\%, CI 0.04-0.07, $P=0.000$ ), wrong indication for drug in 61 incidents $(10.4 \%$, CI $0.08-0.13, P=0.000$ ) and missing information about the drugs in 18 incidents $(3.1 \%$, CI $0.02-0.04, P=0.000)$ (Table 1).

At site 2 (HWP system) approximately 94 incidents (15.5\%, CI 0.13-0.19, $P=0.000)$ showed improper dose, 92 incidents $(15.2 \%$, CI $0.12-0.17, P=0.000)$ of monitoring errors including contraindications and drug interactions, 52 incidents $(8.6 \%, \mathrm{CI} 0.07-0.11, P=0.000)$ of wrong time or schedule of drug administration, 51 incidents $(8.4 \%$, CI $0.07-0.11, P=0.000)$ of no or improper labeling, 53 incidents $(8.7 \%$, CI $0.06-0.1, P=0.000)$ of potential problems with the lack of treatment effectiveness, and 345 other types of incidents $(56.9 \%$, CI 0.6 $0.78, \mathrm{P}=0.000)$ such as missing information about the drugs in 107 incidents (17.7\%, CI 0.14-0.2, $\mathrm{P}=0.000), 68$ incidents $(11.2 \%$, CI $0.08-0.13, \mathrm{P}=0.000)$ of unnecessary medications including many others including product unavailability, medications without need and no entry in records (Table 1 ).

\section{Causes leading towards occurrence of incidents}

Among many causes leading towards incidence occurrence the highly reported cause/s at site 1 (CPOE system) were dose or dosage selection in 233 incidents (39.6\%, CI 0.36-0.44, 0.000), drug selection in 198 incidents $(33.6 \%$, CI $0.3-0.37,0.000)$, human factors in 369 incidents $(62.6 \%, \mathrm{CI} 0.59-0.63, P=0.000)$ which further includes performance deficit of staff in 137 incidents (23.3\%, CI 0.20-0.27, $\mathrm{P}=0.000$ ), Dosage and rate miscalculations in 108 incidents $(18.3 \%$, CI $0.15-0.21, P=$ $0.000)$, transcribing error in 26 incidents $(4.4 \%$, CI 0.03 $0.06, P=0.000)$ and confrontational behavior of the staff in 43 incidents $(7.3 \%, \mathrm{CI} 0.05-0.09, \mathrm{P}=0.000)$. Other causes included lack of monitoring and cross checking practices in 61 incidents $(10.4 \%$, CI $0.08-0.13, \mathrm{P}=0.000)$ and prescribing unnecessary medicines in 30 incidents (5.1\%, CI 0.03-0.07, $P=0.000$ ) (Table 2).
While at site 2 (HWP system) causes leading highly towards incidence occurrence were drug selection in 235 incidents $(38.8 \%$, CI $0.35-0.43,0.000)$, dose or dosage selection in 93 incidents $(15.3 \%$, CI $0.13-0.28,0.000)$, communication barriers caused 58 incidents $(9.7 \%, \mathrm{CI}$ $0.14-0.24, P=0.000$ ), human factors in 438 incidents (72.3\%, CI $0.68-0.75, \mathrm{P}=0.000)$ which further includes performance deficit of staff in 162 incidents $(26.7 \%, \mathrm{CI}$ $0.25-0.33, \quad \mathrm{P}=0.000$ ), confrontational behavior of the staff in 135 incidents $(22.3 \%$, CI $0.18-0.25, \mathrm{P}=0.000)$, transcribing error in 51 incidents $(8.4 \%$, CI $0.06-0.1, P=$ 0.000). Among other causes the most common were lack of monitoring practices i.e. 155 incidents $(25.6 \%$, CI 0.21-0.28, $P=000$ ) (Table 2).

Factors contributing towards the occurrence of incidents Among the contributing factors leading towards occurrence of incidents the highly reported factors at site1 (CPOE system) included staff training in 198 incidents (33.6\%, CI 0.30-0.38, $P=0.000$ ), System covering patient care in 88 incidents $(14.9 \%$, CI $0.12-0.18, P=0.000)$, frequent interruptions in 57 incidents $(9.7 \%$, CI $0.07-0.12$, $\mathrm{P}=0.000$ ) along with other factors in 464 incidents (78.8\%, CI 0.75-0.82, $\mathrm{P}=0.000$ ). Other factors further included highest rate of prescribing practices in 261 incidents $(44.3 \%$, CI $0.40-0.48, P=0.000)$, lack of updated knowledge in 126 incidents $(21.4 \%$, CI $0.18-0.25, P=$ $0.000)$ and staff attitude in 92 incidents $(15.6 \%$, CI 0.14 $0.21, P=0.000)$ as factors which played role in the occurrence of incidents at different levels (Table 3).

At site 2 (HWP system) the presence of factors like staff training in 147 incidents $(24.3 \%$, CI $0.21-0.28, P=$ 0.000 ), system covering patient care in 222 incidents (36.6\%, CI $0.32-0.40, P=0.000$ ), Communication system in 123 incidents $(20.3 \%$, CI $0.17-0.24, P=0.000)$, assignment of inexperienced personnel in 99 incidents $(16.3 \%$, CI $0.13-0.19, P=0.000$ ), policies and procedures in 61 incidents $(10.1 \%$, CI $0.08-0.13, P=0.000)$ along with other factors in 416 incidents $(68.6 \%, 0.65-0.72, P=$ 0.000 ) which lead to the occurrence of these incidents. Among other factors prescribing practices in 248 incidents (40.9\%, CI 0.37-0.45, P = 0.000), staff attitude in 95 incidents $(15.7 \%$, CI $0.13-0.19, \mathrm{P}=0.000)$, lack of interdisciplinary approach in 78 incidents $(12.9 \%$, CI 0.10 $0.16, P=0.000)$, cross checking practice in 57 incidents (9.4\%, CI $0.07-0.12, P=0.000)$ and lack of updated knowledge in 23 incidents $(3.8 \%$, CI $0.02-0.05, \mathrm{P}=0.000)$ resulted in the occurrence of these incidents (Table 3).

\section{Patient outcome}

Patient outcome analysis according to the severity index (NCC-MERP) showed many incidents caused no harm at both sites with a figure of no harm events at site 1 (CPOE system) of 554 incidents (94\%, CI 1.07-1.82, $P=$ 
Table 1 Types of incidents occurring among oncology patients

\begin{tabular}{|c|c|c|c|c|c|c|c|c|c|c|c|c|c|}
\hline \multirow[t]{3}{*}{ Type of incidents } & \multicolumn{6}{|c|}{ Site 1 (CPOE) } & \multicolumn{6}{|c|}{ Site 2 (HWP) } & \multirow[t]{3}{*}{ Total } \\
\hline & \multirow[t]{2}{*}{ No. } & \multirow[t]{2}{*}{$\%$} & \multirow[t]{2}{*}{ Mean } & \multicolumn{2}{|l|}{$95 \% \mathrm{Cl}$} & \multirow{2}{*}{$\begin{array}{l}\text { Sig. } \\
\text { (2-tailed) }\end{array}$} & \multirow{2}{*}{ No. } & \multirow[t]{2}{*}{$\%$} & \multirow[t]{2}{*}{ Mean } & \multicolumn{2}{|l|}{$95 \% \mathrm{Cl}$} & \multirow{2}{*}{$\begin{array}{l}\text { Sig. } \\
\text { (2-tailed) }\end{array}$} & \\
\hline & & & & Lower & Upper & & & & & Lower & Upper & & \\
\hline Dose Omission & 5 & 0.8 & 0.01 & 0.00 & 0.02 & 0.025 & 24 & 4 & 0.04 & 0.02 & 0.05 & 0.000 & 29 \\
\hline Wrong Strength or concentration & 17 & 2.9 & 0.03 & 0.02 & 0.04 & 0.000 & 18 & 3 & 0.03 & 0.02 & 0.05 & 0.000 & 35 \\
\hline Wrong Dosage form & 42 & 7.1 & 0.07 & 0.05 & 0.09 & 0.000 & 25 & 4.1 & 0.04 & 0.03 & 0.06 & 0.000 & 67 \\
\hline Wrong route of administration & 36 & 6.1 & 0.06 & 0.04 & 0.08 & 0.000 & 4 & 0.7 & 0.01 & 0 & 0.01 & 0.000 & 40 \\
\hline Wrong time or schedule & 69 & 11.7 & 0.12 & 0.09 & 0.14 & 0.000 & 52 & 8.6 & 0.09 & 0.07 & 0.11 & 0.000 & 121 \\
\hline Wrong Rate (IV bolus/injection/infusion) & 14 & 2.4 & 0.02 & 0.01 & 0.04 & 0.000 & 28 & 4.6 & 0.05 & 0.03 & 0.07 & 0.000 & 42 \\
\hline Wrong Drug & 116 & 19.7 & 0.20 & 0.16 & 0.23 & 0.000 & 37 & 6.1 & 0.06 & 0.04 & 0.08 & 0.000 & 153 \\
\hline Wrong Technique & 19 & 3.2 & 0.03 & 0.02 & 0.05 & 0.000 & 48 & 7.9 & 0.08 & 0.06 & 0.1 & 0.000 & 67 \\
\hline Wrong Duration & 1 & 0.2 & 0.00 & 0.00 & 0.01 & 0.318 & 17 & 2.8 & 0.03 & 0.02 & 0.04 & 0.000 & 18 \\
\hline Wrong Patient & 1 & 0.2 & 0.00 & 0.00 & 0.01 & 0.318 & - & - & - & - & - & - & 1 \\
\hline Improper Dose & 185 & 31.4 & 0.31 & 0.28 & 0.35 & 0.000 & 94 & 15.5 & 0.16 & 0.13 & 0.19 & 0.000 & 279 \\
\hline Inappropriate diluents & 1 & 0.2 & 0.00 & 0.00 & 0.01 & 0.318 & 10 & 1.7 & 0.02 & 0.01 & 0.03 & 0.002 & 11 \\
\hline Inappropriate Labeling & 12 & 2 & 0.02 & 0.01 & 0.03 & 0.001 & 51 & 8.4 & 0.09 & 0.07 & 0.11 & 0.000 & 63 \\
\hline Monitoring Error (Including Cls*a and $\mathrm{Dls}^{* \mathrm{~b}}$ ) & 86 & 14.6 & 0.15 & 0.12 & 0.17 & 0.000 & 92 & 15.2 & 0.14 & 0.12 & 0.17 & 0.000 & 178 \\
\hline Deteriorated Drug Error & 7 & 1.2 & 0.01 & 0.00 & 0.02 & 0.008 & 1 & 0.2 & 0 & 0 & 0.01 & 0.318 & 8 \\
\hline $\begin{array}{l}\text { Potential problem with the lack of treatment } \\
\text { effectiveness }\end{array}$ & 133 & 22.6 & 0.23 & 0.19 & 0.26 & 0.000 & 53 & 8.7 & 0.08 & 0.06 & 0.1 & 0.000 & 186 \\
\hline Patient suffers or will possibly suffer from ADEs & 69 & 11.7 & 0.12 & 0.09 & 0.14 & 0.000 & 87 & 14.4 & 0.15 & 0.12 & 0.18 & 0.000 & 156 \\
\hline Drug treatment more costly than necessary & 52 & 8.8 & 0.09 & 0.07 & 0.11 & 0.000 & 109 & 18 & 0.18 & 0.14 & 0.21 & 0.000 & 161 \\
\hline Other type of incidents & 229 & 38.9 & 0.39 & 0.35 & 0.43 & 0.000 & 345 & 56.9 & 0.69 & 0.6 & 0.78 & 0.000 & 574 \\
\hline \multicolumn{14}{|l|}{ Other types (breakup) } \\
\hline Unauthorized medication & 12 & 2 & 0.02 & 0.01 & 0.03 & 0.001 & 13 & 2.1 & 0.02 & 0.01 & 0.03 & 0.000 & 25 \\
\hline Replacing medication without approval & 6 & 1 & 0.01 & 0.00 & 0.02 & 0.014 & 1 & 0.2 & 0 & 0 & 0.01 & 0.318 & 7 \\
\hline $\begin{array}{l}\text { Requesting more than required according } \\
\text { to order }\end{array}$ & 15 & 2.5 & 0.03 & 0.01 & 0.04 & 0.000 & 12 & 2 & 0.02 & 0.01 & 0.03 & 0.001 & 27 \\
\hline No entry of drug administration record & - & - & - & - & - & - & 14 & 2.3 & 0.02 & 0.01 & 0.04 & 0.000 & 14 \\
\hline Forgetting to prepare medication & 1 & 0.2 & 0.00 & 0.00 & 0.01 & 0.318 & 20 & 3.3 & 0.03 & 0.02 & 0.05 & 0.000 & 21 \\
\hline Inappropriate storage & & & & & & & 6 & 1 & 0.01 & 0 & 0.02 & 0.014 & 6 \\
\hline Not highlighting administration considerations & 33 & 5.6 & 0.00 & 0.04 & 0.07 & 0.000 & 16 & 2.6 & 0.02 & 0.01 & 0.04 & 0.000 & 49 \\
\hline No entry of patient indication & - & - & - & - & - & - & 7 & 1.2 & 0.01 & 0 & 0.02 & 0.008 & 7 \\
\hline Missing necessary information & 18 & 3.1 & 0.00 & 0.02 & 0.04 & 0.000 & 107 & 17.7 & 0.17 & 0.14 & 0.2 & 0.000 & 125 \\
\hline Unnecessary or medication without need & 11 & 1.9 & 0.06 & 0.01 & 0.03 & 0.001 & 68 & 11.2 & 0.11 & 0.08 & 0.13 & 0.000 & 79 \\
\hline Medication non-compliance & - & - & - & - & - & - & 2 & 0.3 & 0 & 0 & 0.01 & 0.157 & 2 \\
\hline Use of abbreviations & - & - & - & - & - & - & 5 & 0.8 & 0.01 & 0 & 0.01 & 0.025 & 5 \\
\hline Product not available & 20 & 3.4 & 0.00 & 0.02 & 0.05 & 0.000 & 24 & 4 & 0.04 & 0.02 & 0.06 & 0.000 & 44 \\
\hline No drug given for indication & 51 & 8.7 & 0.03 & 0.06 & 0.11 & 0.000 & 33 & 5.4 & 0.06 & 0.04 & 0.08 & 0.000 & 84 \\
\hline Therapeutic duplication & 37 & 6.3 & 0.02 & 0.04 & 0.08 & 0.000 & 20 & 3.3 & 0.03 & 0.02 & 0.05 & 0.000 & 57 \\
\hline Entry of not administered dose in $M A R^{* c}$ & 1 & 0.2 & 0.00 & 0.00 & 0.01 & 0.318 & 3 & 0.5 & 0 & 0 & 0.01 & 0.083 & 4 \\
\hline Incorrect entry in $M A R^{* c}$ & 1 & 0.2 & 0.00 & 0.00 & 0.01 & 0.318 & 5 & 0.8 & 0.01 & 0 & 0.02 & 0.025 & 6 \\
\hline Wrong Indication for drug & 61 & 10.4 & 0.03 & 0.08 & 0.13 & 0.000 & 34 & 5 & 0.06 & 0.04 & 0.08 & 0.000 & 95 \\
\hline Incorrect IV*d to $\mathrm{PO}^{* e}$ conversion & 1 & 0.2 & 0.09 & 0.00 & 0.01 & 0.318 & 9 & 1.5 & 0.02 & 0.01 & 0.03 & 0.003 & 10 \\
\hline Total & 1362 & - & - & - & - & - & 1494 & - & - & - & - & - & 2856 \\
\hline
\end{tabular}


Table 2 Causes leading towards occurrence of incidents

\begin{tabular}{|c|c|c|c|c|c|c|c|c|c|c|c|c|c|}
\hline \multirow[t]{3}{*}{ Causes of incidents } & \multicolumn{6}{|c|}{ Site 1 (CPOE) } & \multicolumn{6}{|c|}{ Site 2 (HWP) } & \multirow[t]{3}{*}{ Total } \\
\hline & \multirow[t]{2}{*}{ No. } & \multirow[t]{2}{*}{$\%$} & \multirow[t]{2}{*}{ Mean } & \multicolumn{2}{|l|}{$95 \% \mathrm{Cl}$} & \multirow{2}{*}{$\begin{array}{l}\text { Sig. } \\
\text { (2-tailed) }\end{array}$} & \multirow{2}{*}{ No. } & \multirow[t]{2}{*}{$\%$} & \multirow[t]{2}{*}{ Mean } & \multicolumn{2}{|c|}{$95 \% \mathrm{Cl}$} & \multirow{2}{*}{$\begin{array}{l}\text { Sig. } \\
\text { (2-tailed) }\end{array}$} & \\
\hline & & & & Lower & Upper & & & & & Lower & Upper & & \\
\hline Communication & 21 & 3.5 & 0.04 & 0.02 & 0.06 & 0.000 & 58 & 9.7 & 0.19 & 0.14 & 0.24 & 0.000 & 79 \\
\hline Name and Sound confusion & - & - & - & - & - & - & 1 & 0.2 & 0.00 & 0.00 & 0.01 & 0.318 & 1 \\
\hline Labeling & 8 & 1.4 & 0.01 & 0.00 & 0.02 & 0.005 & 28 & 4.6 & 0.05 & 0.03 & 0.07 & 0.000 & 36 \\
\hline Packaging or design & 6 & 1 & 0.01 & 0.00 & 0.02 & 0.014 & - & - & - & - & - & - & 6 \\
\hline Drug selection & 198 & 33.6 & 0.34 & 0.3 & 0.37 & 0.000 & 235 & 38.8 & 0.39 & 0.35 & 0.43 & 0.000 & 433 \\
\hline Inappropriate drug form & 54 & 9.2 & 0.09 & 0.07 & 0.12 & 0.000 & 27 & 4.5 & 0.05 & 0.03 & 0.06 & 0.000 & 81 \\
\hline Dose or dosage selection & 233 & 39.6 & 0.40 & 0.36 & 0.44 & 0.000 & 93 & 15.3 & 0.15 & 0.13 & 0.28 & 0.000 & 326 \\
\hline Inappropriate duration of therapy & 15 & 2.5 & 0.03 & 0.01 & 0.04 & 0.000 & 27 & 4.5 & 0.05 & 0.03 & 0.06 & 0.000 & 42 \\
\hline DRP*a with drug use in spite of instruction & - & - & - & - & - & - & 6 & 1 & 0.02 & 0.00 & 0.03 & 0.033 & 6 \\
\hline $\mathrm{DRP}^{* a}$ related to logistics & 2 & 0.3 & 0.00 & 0.00 & 0.01 & 0.157 & 66 & 10.9 & 0.10 & 0.08 & 0.13 & 0.000 & 68 \\
\hline $\begin{array}{l}\mathrm{DRPs}^{* a} \text { related to the patient personality } \\
\text { or behavior }\end{array}$ & 7 & 1.2 & 0.00 & 0.00 & 0.01 & 0.008 & 7 & 1.2 & 0.01 & 0.00 & 0.02 & 0.008 & 14 \\
\hline Human factors (breakup) & 369 & 62.6 & 0.63 & 0.59 & 0.67 & 0.000 & 438 & 72.3 & 0.72 & 0.68 & 0.75 & 0.000 & 807 \\
\hline Knowledge deficit & 55 & 9.3 & 0.09 & 0.07 & 0.12 & 0.000 & 57 & 9.4 & 0.09 & 0.07 & 0.11 & 0.000 & 112 \\
\hline Performance deficit & 137 & 23.3 & 0.23 & 0.20 & 0.27 & 0.000 & 162 & 26.7 & 0.29 & 0.25 & 0.33 & 0.000 & 299 \\
\hline Dosage/rate Miscalculations & 108 & 18.3 & 0.18 & 0.15 & 0.21 & 0.000 & 42 & 6.9 & 0.08 & 0.05 & 0.1 & 0.000 & 150 \\
\hline System error & 7 & 1.2 & 0.01 & 0.00 & 0.02 & 0.008 & 5 & 0.8 & 0.01 & 0.00 & 0.02 & 0.025 & 12 \\
\hline Error in stocking & - & - & - & - & - & - & 28 & 4.6 & 0.05 & 0.03 & 0.06 & 0.000 & 28 \\
\hline Drug preparation or dilution error & 5 & 0.8 & 0.01 & 0.00 & 0.02 & 0.025 & 14 & 2.3 & 0.02 & 0.01 & 0.03 & 0.000 & 19 \\
\hline Transcription error & 26 & 4.4 & 0.04 & 0.03 & 0.06 & 0.000 & 51 & 8.4 & 0.08 & 0.06 & 0.1 & 0.000 & 77 \\
\hline High Volume workload & 40 & 6.8 & 0.07 & 0.05 & 0.09 & 0.000 & 42 & 6.9 & 0.07 & 0.05 & 0.09 & 0.000 & 82 \\
\hline Fatigue or lack of sleep & - & - & - & - & - & - & 8 & 1.3 & 0.01 & 0.00 & 0.02 & 0.005 & 8 \\
\hline Confrontational or intimidating behavior & 43 & 7.3 & 0.07 & 0.05 & 0.09 & 0.000 & 135 & 22.3 & 0.21 & 0.18 & 0.25 & 0.000 & 178 \\
\hline Others (breakup) & 115 & 19.5 & 0.20 & 0.16 & 0.23 & 0.000 & 247 & 40.8 & 0.40 & 0.36 & 0.44 & 0.000 & 362 \\
\hline $\begin{array}{l}\text { Lack of monitoring or cross checking } \\
\text { practices }\end{array}$ & 61 & 10.4 & 0.10 & 0.08 & 0.13 & 0.000 & 155 & 25.6 & 0.25 & 0.21 & 0.28 & 0.000 & 216 \\
\hline Lack of all-time oncology pharmacist & 14 & 2.4 & 0.02 & 0.01 & 0.04 & 0.000 & 44 & 7.3 & 0.07 & 0.05 & 0.09 & 0.000 & 58 \\
\hline Less knowledge of antibiotic stewardship & 27 & 4.6 & 0.05 & 0.03 & 0.06 & 0.000 & 18 & 3 & 0.03 & 0.02 & 0.05 & 0.000 & 45 \\
\hline Unnecessary medicine/s & 30 & 5.1 & 0.05 & 0.03 & 0.07 & 0.000 & 45 & 7.4 & 0.07 & 0.05 & 0.1 & 0.000 & 75 \\
\hline Wrong drug combination/s & 20 & 3.4 & 0.03 & 0.02 & 0.05 & 0.000 & 3 & 0.5 & 0.01 & 0.00 & 0.01 & 0.083 & 23 \\
\hline Total & 1601 & & & & & & 2042 & & & & & & 3643 \\
\hline
\end{tabular}

$0.000)$ and 376 incidents $(62 \%, \mathrm{CI} 1.41-1.61, P=0.000)$ at site 2 (HWP system). Among no harm events the Category $\mathrm{C}$ events were highly reported at site 1 (CPOE system) with an occurrence of 334 incidents (56.7\%) and Category D events were highly reported at site 2 (HWP system) with an occurrence of 189 incidents (31.2\%).

The events which caused harm were high in occurrence at site 2 (HWP system) with an incidence of 221 (36.5\%, CI $0.50-0.63, \mathrm{P}=0.000)$ and at site 1 (CPOE system) an incidence of $21(3.6 \%$, CI $0.02-0.05, \mathrm{P}=0.000)$. The Category $\mathrm{E}$ events were reported highly at site 1 (CPOE system) with an occurrence of 21 incidents (3.6\%) and 115 incidents (19\%) at site 2 (HWP system).
While occurrence of Category $F$ events reported were 90 incidents (14.9\%), and Category G events reported were 15 incidents $(2.5 \%)$ only at site 2 (HWP system) (Table 3 ).

Settings and stages of incidents occurrence and discovery At site 1 (CPOE system) setting of initial incident showed greater incidence at the level of ward in 287 incidents $(48.7 \%)$ and prescribers' office in 285 incidents (48.4\%). Site 2 (HWP system) also showed greater incidence of setting of initial incident at the level of ward in 294 incidents (48.5\%) and at prescribers' office in 269 incidents (44.4\%) (Table 4). 
Table 3 Factors contributing towards the occurrence of incidents and their severity index

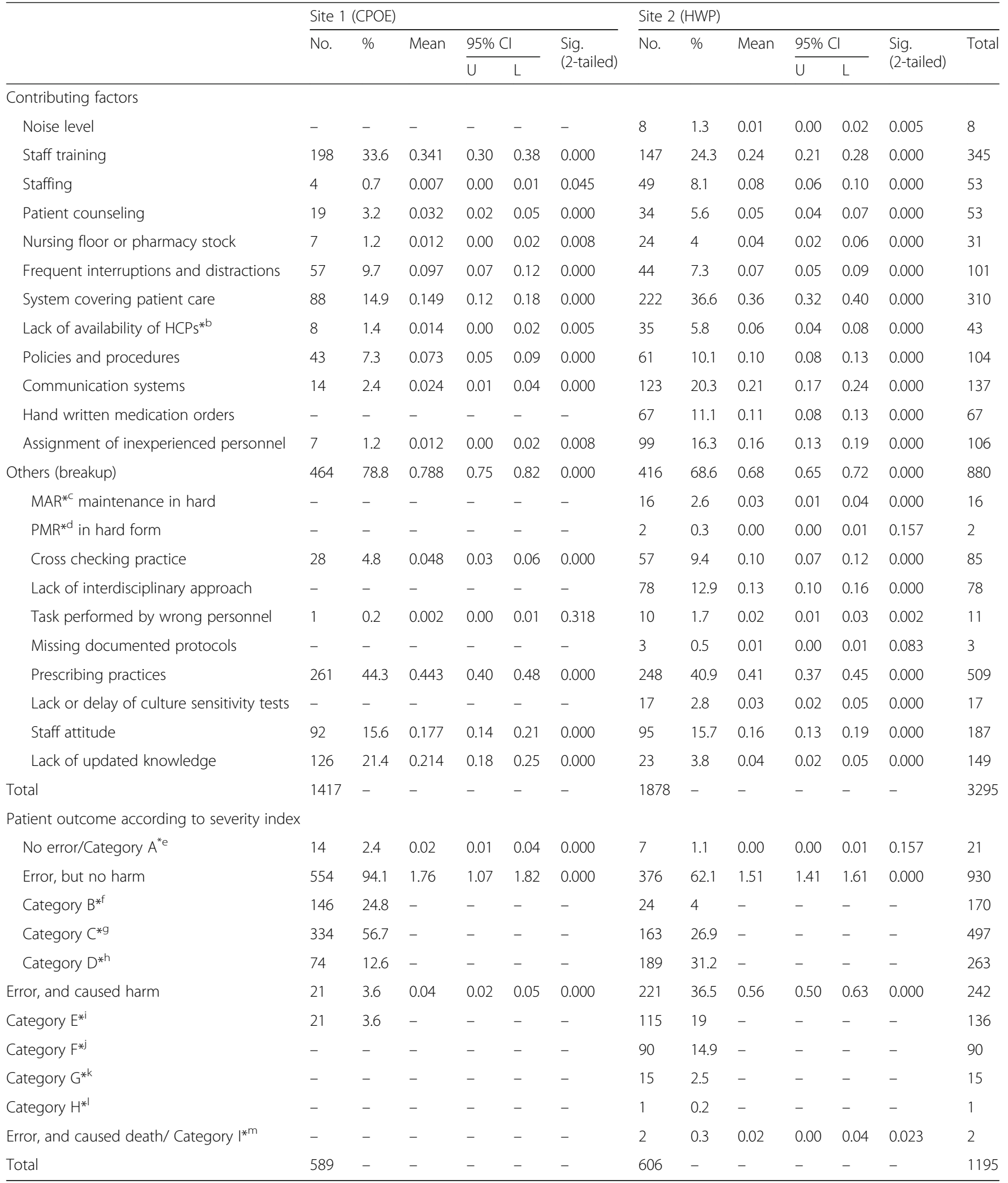

$D R P S^{* a}$ Drug related problems, $H C P S^{* \mathrm{~b}}$ Health care professionals, $M A R^{* c}$ Medication administration record, $P M R^{* \mathrm{~d}}$ Patient medication record. Category $\mathrm{A}^{*}$ : Circumstances or events that have the capacity to cause error, Category $B^{* f}:$ An error occurred but the error did not reach the patient, Category $C^{* 9}$ : An error occurred that reached the patient, but did not cause patient harm, Category $D^{* h}$ : Error occurred, reached patient, require monitoring to ensure no harm is occurred and/or require intervention to prevent harm, Category $\mathrm{E}^{* 1}$ : Error occurred, resulting or contributing to temporary harm to the patient, requiring intervention, Category $\mathrm{F}^{* j}$ : Error occurred, contributing to or resulting in temporary harm to the patient, and requiring initial or prolongation of hospitalization, Category $\mathrm{G}^{* \mathrm{k}}$ : Error occurred, contributing to resulting in permanent patient harm, Category $\mathrm{H}^{*}$ : Error occurred, requiring intervention to sustain life, and Category $I^{* m}:$ Error occurred, resulting in death of the patient 
Table 4 Rate of incidents at different settings and stages; their preventability and interventions

\begin{tabular}{|c|c|c|c|c|c|c|}
\hline \multirow[t]{2}{*}{ Variable } & \multicolumn{2}{|c|}{ Site 1 (CPOE) } & \multicolumn{2}{|c|}{ Site 2 (HWP) } & \multicolumn{2}{|l|}{ Total } \\
\hline & $\mathrm{N}$ & $\%$ & $\mathrm{~N}$ & $\%$ & $\mathrm{~N}$ & $\%$ \\
\hline \multicolumn{7}{|l|}{ Setting of initial incidents } \\
\hline Oncology ward & 287 & 48.7 & 294 & 48.5 & 581 & 97.2 \\
\hline Pharmacy & 12 & 2 & 28 & 4.6 & 40 & 6.6 \\
\hline Prescriber's Office & 285 & 48.4 & 269 & 44.4 & 554 & 92.8 \\
\hline Patient home & - & - & 10 & 1.7 & 10 & 1.7 \\
\hline Other & 5 & 0.8 & 5 & 0.8 & 10 & 1.6 \\
\hline \multicolumn{7}{|l|}{ Stages of initial error } \\
\hline Physician & 516 & 87.6 & 495 & 81.7 & 1011 & 169.3 \\
\hline Consultant & 372 & 63.2 & 389 & 64.2 & 761 & 127.4 \\
\hline Medical Officer & 139 & 23.8 & 96 & 15.8 & 235 & 39.6 \\
\hline Fellow & - & - & 10 & 1.7 & 10 & 1.7 \\
\hline Resident & 5 & 0.6 & - & - & 5 & 0.6 \\
\hline Pharmacist & 39 & 6.6 & 21 & 3.5 & 56 & 10.1 \\
\hline Prescription receiving or checking & 29 & 4.9 & 15 & 2.5 & 43 & 7.4 \\
\hline Prescription filling & 9 & 1.5 & 6 & 1 & 12 & 2.5 \\
\hline Dispensing & 1 & 0.2 & - & - & 1 & 0.2 \\
\hline Nurse & 25 & 4.3 & 75 & 12.3 & 100 & 16.6 \\
\hline Medication received & 24 & 4.1 & 62 & 10.2 & 86 & 14.3 \\
\hline Administered & 1 & 0.2 & 13 & 2.1 & 14 & 2.3 \\
\hline Patient Care Giver & 14 & 2.4 & 5 & 0.8 & 19 & 3.2 \\
\hline Other & 2 & 0.3 & 4 & 0.7 & 6 & 1 \\
\hline Support personnel & 2 & 0.3 & - & - & 2 & 0.3 \\
\hline Health Profession Students & - & - & 1 & 0.2 & 1 & 0.2 \\
\hline Physician assistant & - & - & 3 & 0.5 & 3 & 0.5 \\
\hline Unknown & - & - & 10 & 1.7 & 10 & 1.7 \\
\hline \multicolumn{7}{|l|}{ Stage of error discovery } \\
\hline Physician & 48 & 8.1 & 24 & 4 & 72 & 12.1 \\
\hline Consultant & 29 & 4.9 & - & - & 29 & 4.9 \\
\hline Medical Officer & 19 & 3.2 & 13 & 2.1 & 32 & 5.3 \\
\hline Fellow & - & - & 11 & 1.8 & 11 & 1.8 \\
\hline Resident & - & - & - & - & - & - \\
\hline Pharmacist & 482 & 81.8 & 14 & 2.3 & 496 & 84.1 \\
\hline Prescription receiving or checking & 378 & 64.2 & 11 & 2.1 & 389 & 66.3 \\
\hline Prescription filling & 90 & 15.3 & 3 & 0.5 & 93 & 15.8 \\
\hline Dispensing & 14 & 2.4 & - & - & 14 & 2.4 \\
\hline Nurse & 15 & 2.6 & 30 & 5 & 45 & 7.6 \\
\hline Medication received & 8 & 1.4 & 24 & 4 & 32 & 5.4 \\
\hline Administered & 7 & 1.2 & 6 & 1 & 13 & 2.2 \\
\hline Patient care giver & - & - & 1 & 0.2 & 1 & 0.2 \\
\hline Other & 44 & 7.5 & 536 & 88.4 & 580 & 95.9 \\
\hline Health Profession Students & - & - & 9 & 1.5 & 9 & 1.5 \\
\hline Support personnel & 1 & 0.2 & - & - & 1 & 0.2 \\
\hline
\end{tabular}


Table 4 Rate of incidents at different settings and stages; their preventability and interventions (Continued)

\begin{tabular}{|c|c|c|c|c|c|c|}
\hline \multirow[t]{2}{*}{ Variable } & \multicolumn{2}{|c|}{ Site 1 (CPOE) } & \multicolumn{2}{|c|}{ Site 2 (HWP) } & \multicolumn{2}{|l|}{ Total } \\
\hline & $\mathrm{N}$ & $\%$ & N & $\%$ & $\mathrm{~N}$ & $\%$ \\
\hline Physician Assistant & 1 & 0.2 & - & - & 1 & 0.2 \\
\hline Primary Investigator (PI) & 42 & 7.1 & 527 & 86.9 & 569 & 94 \\
\hline Unknown & - & - & 1 & 0.2 & 1 & 0.2 \\
\hline \multicolumn{7}{|c|}{ Rate of MEs ${ }^{\mathrm{a}}$ without harm and potential ADEs ${ }^{\mathrm{b}}$} \\
\hline MEs without harm & 574 & 97.5 & 20 & 3.3 & 594 & 100.8 \\
\hline Potential ADEs & 15 & 2.5 & 586 & 96.7 & 601 & 99.2 \\
\hline \multicolumn{7}{|l|}{ Preventability of the event } \\
\hline Preventability & 582 & 98.8 & 601 & 99.2 & 1183 & 198 \\
\hline Not-preventable & 7 & 1.2 & 5 & 0.8 & 12 & 2 \\
\hline Total & 589 & 100 & 606 & 100 & 1195 & \\
\hline \multicolumn{7}{|l|}{ Intervention } \\
\hline Medical intervention & 533 & 90.5 & 48 & 7.9 & 581 & 98.4 \\
\hline
\end{tabular}

Stages of initial incident occurrence showed higher incidence at the level of physician at incident rate of 516 (87.6\%), pharmacist at incident rate of 39 (6.6\%), nurse at incident rate of $25(4.3 \%)$, patient care giver at incident rate of $14(2.4 \%)$ and others at incident rate of 2 $(0.3 \%)$ at site 1 (CPOE system). At site 2 (HWP system) initial error showed higher occurrence at the level of physician at incident rate of 495 (81.7\%), pharmacist at incident rate of 21 (3.5\%) followed by nurse at incident rate of 75 (12.3\%), patient care giver at incident rate of 5 $(0.8 \%)$ and others at incident rate of $4(0.7 \%)$ (Table 4$)$.

Stages of incident discovery showed a greater percentage of discovery and interception at the level of pharmacy at site 1 (CPOE system) at a rate of 482 incidents (81.8\%) followed by physician at a rate of 48 incidents (8.1\%), nursing at a rate of 15 incidents $(2.6 \%)$ and others at a rate of 44 incidents $(7.5 \%)$. At site 2 ((HWP system) the primary investigator among others (536 incidents, $88.4 \%$ ) discovered and intercepted greater number of incidents at a rate of 536 incidents (86.9\%) followed by nursing at a rate of 30 incidents (5\%) and pharmacy at a rate of 14 incidents $(2.3 \%)$ (Table 4$)$.

\section{Rate of incidents, preventability and interventions}

The medication review process identified incidence rate of $574(97.5 \%)$ medication errors without harm at site 1 (CPOE system) and $20(3.3 \%)$ at site 2 (HWP system) among all reported incidents. The preventability analysis showed a rate of prevention of 582 incidents $(98.8 \%)$ and 7 incidents $(1.2 \%)$ were not preventable at site 1 (CPOE system). At site 2 (HWP system) 601 incidents (99.2\%) showed preventability and 5 incidents $(0.8 \%)$ were not preventable.

Medical interventions were done and reported at site 1 (CPOE system) by all HCPs involved with an incidence rate of 533 incidents (90.5\%) as compared to site 2 (HWP system) with an incidence rate of 48 incidents (8\%) (Table 4).

\section{Medication classes involved}

The medication classes involved in the occurrence of medication related events reported at site 1 (CPOE system) highly reported antibiotics in 259 incidents (44\%), analgesics and antipyretics (NSAIDs and Opiates) in 67 incidents (11.4\%), and antiemetic in 44 incidents (7.5\%) along with other classes including antihistamines, antineoplastic agents, anti-diabetic agents, coagulants and drugs acting on central nervous system. At site 2 (HWP system) the medication classes highly reported included antiemetic in 96 incidents (15.8\%), antibiotics in 77 incidents (12.7\%), dietary supplements in 72 incidents (11.9\%), antineoplastic agents in 57 incidents (9.4\%), analgesics and antipyretics (NSAIDs and Opiates) in 55 incidents $(9.1 \%)$ along with other classes like replacement solutions, human albumin, coagulants and cathartics and laxatives (Table 5).

\section{Discussion}

This study focused on the incidence of medication related events among cancer in-patients along with the concurrent evaluation of causes and system related factors at different levels responsible for their occurrence. Like the studies done in the past by Watts et al. [15] and Jayanti et al. [8] this study showed that the most common types of incidents reported at both sites included improper dose, monitoring errors, wrong time or schedule of administration and many other types like drug labelling, missing drug related information, dose prescribing and/or dispensing incidents. At the setting with computerized prescribing system (CPOE) the 
Table 5 Medication classes involved in occurrence of incidents

\begin{tabular}{|c|c|c|c|c|c|c|}
\hline \multirow[t]{2}{*}{ Medication classes } & \multicolumn{2}{|c|}{ Site 1 (CPOE) } & \multicolumn{2}{|c|}{ Site 2 (HWP) } & \multicolumn{2}{|l|}{ Total } \\
\hline & $\mathrm{N}$ & $\%$ & $\mathrm{~N}$ & $\%$ & N & $\%$ \\
\hline Analgesics and antipyretics (NSAIDs ${ }^{* a}+$ Opiates) & 67 & 11.4 & 55 & 9.1 & 122 & 20.5 \\
\hline Antacids & 31 & 5.3 & 42 & 6.9 & 73 & 12.2 \\
\hline Antibiotics or antibacterial agents & 259 & 44 & 77 & 12.7 & 336 & 56.7 \\
\hline Anticonvulsants & 1 & 0.2 & 1 & 0.2 & 2 & 0.4 \\
\hline Antidepressants + Tranquilizers & 4 & 0.7 & 1 & 0.2 & 5 & 0.9 \\
\hline Anti-diabetic agents (Insulin and sulfonylureas) & 10 & 1.7 & 4 & 0.7 & 14 & 2.4 \\
\hline Anti-diarrheal agents & - & - & 2 & 0.3 & 2 & 0.3 \\
\hline Antiemetic & 44 & 7.5 & 96 & 15.8 & 140 & 23.3 \\
\hline Anti-flatulent & - & - & 2 & 0.3 & 2 & 0.3 \\
\hline Antihistamines & 23 & 3.9 & 13 & 2.1 & 36 & 6 \\
\hline Antineoplastic agents & 17 & 2.9 & 57 & 9.4 & 74 & 12.3 \\
\hline Antiprotozoal + Anti-leprosy agents & 14 & 2.4 & 4 & 0.7 & 18 & 3.1 \\
\hline Antiviral & - & - & 3 & 0.5 & 3 & 0.5 \\
\hline Appetite stimulants & - & - & 5 & 0.8 & 5 & 0.8 \\
\hline Benzodiazepine antagonists & 4 & 0.7 & - & - & 4 & 0.7 \\
\hline Bisphosphonates & - & - & 8 & 1.3 & 8 & 1.3 \\
\hline Blood derivatives & - & - & 2 & 0.3 & 2 & 0.3 \\
\hline $\begin{array}{l}\text { Bronchodilators + Immunosuppressive agents + Mast cell } \\
\text { stabilizers + Xanthine oxidase inhibitors }\end{array}$ & 1 & 0.2 & - & - & 1 & 0.2 \\
\hline Cardiac drug + ACEls $^{* \mathrm{~b}}+$ Calcium antagonists + Cardiac glycosides & - & - & 4 & 0.7 & 4 & 0.7 \\
\hline Cathartics/Laxatives & 13 & 2.2 & 13 & 2.1 & 26 & 4.3 \\
\hline $\begin{array}{l}\text { CNS depressants + Sedatives, hypnotics and Anxiolytics } \\
\text { (Barbiturates and Benzodiazepines) }\end{array}$ & 10 & 1.7 & 24 & 4 & 34 & 5.7 \\
\hline Coagulants and Anticoagulants & 19 & 3.2 & 8 & 1.3 & 27 & 4.5 \\
\hline Colony stimulating factor (CSF) & 9 & 1.5 & 5 & 0.8 & 14 & 2.3 \\
\hline Cyto-protectants (Mesna) & - & - & 6 & 1 & 6 & 1 \\
\hline Devices & - & - & 2 & 0.3 & 2 & 0.3 \\
\hline Dietary supplements and Vitamins & 7 & 1.2 & 72 & 11.9 & 79 & 13.1 \\
\hline Diuretics & - & - & 11 & 1.8 & 11 & 1.8 \\
\hline Enzymes & - & - & 1 & 0.2 & 1 & 0.2 \\
\hline Expectorants & - & - & 5 & 0.8 & 5 & 0.8 \\
\hline Human albumin & - & - & 7 & 1.2 & 7 & 1.2 \\
\hline Immunomodulatory agents & - & - & 3 & 0.5 & 3 & 0.5 \\
\hline Monoclonal antibodies & - & - & 5 & 0.9 & 5 & 0.9 \\
\hline Mouth washes and gargles & 12 & 2 & - & - & 12 & 2 \\
\hline $\begin{array}{l}\text { Ointments/Solutions/Suspensions ophthalmic + } \\
\text { Otic/Ophthalmic/Nasal preparations }\end{array}$ & 7 & 1.2 & 4 & 0.7 & 11 & 1.9 \\
\hline Opiates & - & - & 1 & 0.2 & 1 & 0.2 \\
\hline Para-sympatholytic agents & 4 & 0.7 & - & - & 4 & 0.7 \\
\hline Quinolones & - & - & 3 & 0.5 & 3 & 0.5 \\
\hline Replacement solutions/ORS*c/Minerals/Elements/Electrolytes & 16 & 2.7 & 24 & 4 & 40 & 6.7 \\
\hline Skeletal muscle relaxants & 4 & 0.7 & - & - & 4 & 0.7 \\
\hline Steroids & 4 & 0.7 & 11 & 1.8 & 15 & 2.5 \\
\hline Sugar/Salt substitutes & 2 & 0.3 & 14 & 2.3 & 16 & 2.6 \\
\hline
\end{tabular}


Table 5 Medication classes involved in occurrence of incidents (Continued)

\begin{tabular}{|c|c|c|c|c|c|c|}
\hline \multirow[t]{2}{*}{ Medication classes } & \multicolumn{2}{|c|}{ Site 1 (CPOE) } & \multicolumn{2}{|c|}{ Site 2 (HWP) } & \multicolumn{2}{|c|}{ Total } \\
\hline & $\bar{N}$ & $\%$ & $\bar{N}$ & $\%$ & $\bar{N}$ & $\%$ \\
\hline Sympathomimetic agents & 7 & 1.2 & 2 & 0.3 & 9 & 1.5 \\
\hline Thrombolytic agents & - & - & 4 & 0.7 & 4 & 0.7 \\
\hline Uricosuric agents & - & - & 3 & 0.5 & 3 & 0.5 \\
\hline Total & 589 & 100 & 606 & 100 & 1195 & \\
\hline
\end{tabular}

${ }^{\mathrm{a}}$ NSAIDs Non-steroidal anti-inflammatory drugs, ${ }^{\mathrm{b}}$ ACEls Angiotensin converting enzyme inhibitors and ${ }^{\mathrm{c}}$ ORS Oral rehydrating salts

incidence of reporting any medication related event was high and that's why most of the errors were intercepted before reaching the patients. The electronic system made it possible to analyze each medication order at several points. As compared to the electronic system the setting with hand written prescribing and electronic transcribing with only limited access to medication related data several types of incidents like labeling instructions, drug dilution and weight based dosing protocols, unnecessary medications resulting in increase in treatment cost and polypharmacy occurred frequently and many went unreported. Watts et al. showed greater incidence of similar errors including dosing errors (42\%), roadmap errors (26\%) and timing errors (12\%) [16]. Another study by Jayanti et al. showed comparing rates of prescription error types including missing information (47.1\%) and abbreviations in pre-medication (23.3\%). It showed potential errors like incomplete premedication (32.7\%), dosing errors in anti-cancer drugs (3\%), missing dosage forms $(1.2 \%)$, missing information on diluents (3.8\%) and time of infusion (34.9\%) [9].

Among causes the major cause was human factors resulting in the occurrence of medication related events. Human factors including performance deficit, miscalculations of dosage and/or infusion rates, behavior of HCPs were the major causes of medication related events may lead to MEs or potential ADEs. At hand written setting heavy work load and lack of staff were the major causes that resulted in errors because there is no cross check point which can analyze every order. The lack of interdisciplinary approach subjecting nursing staff to prepare medication at bed side further increases the chance of error at any setting. Electronic system at site 1 with CPOE and CDSS greatly helped out in reduction of manual workload. The medication preparation and dilution at pharmacy further reduced the chances of drug dose calculations and concentrations. As discussed by Jayanthi et al. our study also looked for the factors contributing in the occurrence of medication related incidents. Staff training in the specialty of chemotherapy and critical care, staff replacements by specialized staff for chemotherapy, supervision of residents and nursing students, improvement in the system for patient care, updating and implementing electronic system according to present requirements are the factors which will bring a great difference in the occurrence and interception of medication errors. Jayanthi et al. described similar aspects in their study like time limitations, interruptions, lack of knowledge and attention paid to drug dosing and preparation resulting in a number of errors. The limitation of both studies (at site 2 with HWP in our study) was also same as the patient profile was mostly incomplete lacking basic medical and/or medication information of the patient [11]. Watts and Parsons studied the effect of pharmacist driven intervention which showed a $50 \%$ decrease in the rate of medication errors indicating that the pharmacist evaluation greatly reduces reaching of medication error to the patient [15]. Al-Dawailie in his practice report described that a huge variety of errors were associated with hand written prescribing practice as our study reported at site 2 with HWP [17].

Our study provided a detailed overview of severity of all medication related events including medication errors without harm and the potential ADEs which are mostly not done in many studies. This study categorized outcome on the basis of medication error index presented by NCC-MERP. Our study showed that at the setting working on CPOE/CDSS electronic system most of the incidents are intercepted before reaching the patient. Many errors which reached the patients were intercepted by the pharmacist's rounds along with medical practitioners at each unit. During ward rounds and during medication review electronically medical interventions were done and discussed with the practitioners which if approved was applied. While at the setting with a limited access to drug databases a lot of incidents reached the patients without interception. A large number of incidents reached the patients which needed evaluation for harm. This lack of updated system and least involvement of clinical pharmacist during ward rounds and during dispensing from pharmacies reduced the chances of medical interventions which could lead to permanent patient harm, increasing length of stay, and medication cost. Ranchon et al. provided almost similar results on the severity of outcomes. The study suggests that the toxic nature of cytotoxic drugs, patient condition 
and comorbidities, complex regimens and frequent dose adjustments and calculations makes the outcomes severe. The outcome evaluation though requires close observation which may affect the results of such studies.

The errors were highly reported at the level of prescription at prescriber's office and oncology ward. Error discovery was higher at pharmacy level at site 1 working on electronic system with CPOE and CDSS as compared to site 2 with most of the work done manually and least updated electronic system. It elucidates that pharmacy department is efficiently involved in the medication utilization review process at site 1 with complete access to patient medication and medical record based electronic system which makes it easier to detect any medication related event as compared to site 2 . The pharmacist's involvement during medication use process greatly reduces the occurrence of medication errors in chemotherapy patients. Ranchon et.al showed an incidence of $5.2 \%$ prescription errors in cancer patients which is quite low as compared to the rate at site 2 in our study and almost equal to site 1 . The hospital reported by Ranchon et al. had a same electronic system as at our study's site 2 and a handwritten prescription approach but the prescriptions were sent to pharmacy department before administering to the patients for complete review which greatly reduced the number of medication errors reaching the patient. The study showed a low percentage of administration $(0.02 \%)$ and dispensing $(0.16 \%)$ errors as compared to our study. Both; our study and the Ranchon et al. study; has underreported errors at administration and pharmacy level due to blame and shame approach and lack of time and observation strategies. Watts et al. showed similar results while studying chemotherapy medication errors at a pediatric hospital at a low rate of $6 / 1000$ patient visits while 3.9/1000 dispensing orders. After implementation of a pharmacy surveillance system the error rate was further reduced by $50 \%$ at both ends. The study focused on the need of multidisciplinary review of policies and procedures to ensure patient safety [15].

Our study found a higher incidence of medication related events (potential ADEs) at site 2 (HWP system) while rate of medication errors without harm and interventions was reported highly at site 1 . The higher incidence of potential ADEs at site 2 might be due to the absence of clinical pharmacists, lack of staff training, less updated software and communication among HCPs. Errors nature was preventable mostly concluding that proper cross checking could prevent a greater incidence before reaching the patient.

Drugs highly at risk of medication errors in our study were antibiotics the most commonly prescribed class of drugs in our health care settings, anti- analgesics and antipyretics supplements, antacids, anti-cancer drugs, and electrolytes because of less consideration while prescribing these classes of drugs. Tang et al. found similar results in his study. Antibiotics were the leading class of drugs (38.9\%) subjected to errors due to its prescribing pattern and confusion with other drugs having similar names. Other drugs reported were analgesics (6.9\%), electrolytes $(8.4 \%)$, antidiabetics $(6.9 \%)$ and other classes (38.9\%). Ranchon et al. predictors of prescribing errors among cancer patients showed a 3.15\% error rate among all antineoplastic prescriptions [13].

A detailed study by Richard J. Fitz Gerald showed the importance of accurate medication and medical history in the prevention of medication errors. Errors were more common when patients admitted at hospitals because of incomplete medication histories. Many drugs can cause or mask many conditions like preventing tachycardia by beta adrenoceptor antagonists in hemorrhagic patients, abdominal pain by corticosteroids in perforated duodenal ulcers and/or masking the effects of investigations as thyroid function tests by amiodarone. It also showed that to prevent such errors pharmacists can perform much better roles as compared to physicians and nurses due to their ability to take detailed medication histories and compliance records [16].

\section{Conclusion}

Our study revealed many issues of concern at cancer care settings working on different systems. CPOE with CDSS will greatly reduce the occurrence of medication errors by decreasing the workload of every member involved in pharmaceutical care. Regular staff training and incorporation of pharmacist at different checkpoints will improve health care provision. Regular root cause analysis by a health care team will greatly improve the system making better utilization of resources. The limitation of this study was the less number of data collection members. The handwritten prescriptions, lack of ward pharmacists, under-reporting of mediation errors and time constraints at site 2 may affect the actual number of errors.

\section{Study strengths}

This study has focused on the root causes and contributing factors in two systems working on different approaches of prescribing, dispensing and administration. The incidence and prevalence of medication related incidents are mostly studied without considering the factors involved which are leading to their occurrence. For improvement in our health system and patient safety more studies like this are needed to be done on a broader level.

\section{Limitations}

The limitations of this study includes 
- Time limit as the study was done only for few months. It is needed to be done for a period of a year at least to better understand the occurrence rates of medication related events.

- The sample size needed to be increased which was limited because of lack of data collection personnel.

- All the health care team members including prescribers, pharmacists and nurses are need to be a part of such research team to avoid any biasness and misinterpreting any medication order.

- Many of the events may go un-reported due to lack of personnel.

- A more strong statistical analysis is needed for handling such a huge data with so many variables.

\section{Abbreviations}

ADEs: Adverse drug events; BNF: British National Formulary;

CDSS: Computerized decision support system; Cl: Confidence interval; CPOE: Computerized Prescription Order Entry; HCPs: Health care professionals; HWP: Hand written prescription; MEs: Medication errors; NCCMERP: National Coordinating Center for Medication Error Reporting and Prevention; NCCN: National Comprehensive Cancer Network

\section{Acknowledgements}

All the medical practitioners and hospital staff who made this research possible are acknowledged.

\section{Authors' contributions}

MA performed the data collection, analyzed and interpreted the data regarding the medication incidents. MA contributed a major part in writing manuscript. AK provided the supervisory services and plotting the conception of research. TMK contributed in plotting the research concept, the research analysis and publication of the manuscript. MK was a part during data analysis. All authors have read and approved the manuscript and there is no conflict of interest among them.

\section{Funding}

Not applicable.

\section{Availability of data and materials}

The datasets used and/or analyzed during the current study are available from the corresponding author on reasonable request.

\section{Ethics approval and consent to participate}

Ethical approval was obtained from the Bioethical Committee of the institute (Quaid I Azam University Islamabad). Further the Rehman Medical InstituteResearch Ethics Committee and the senior management officer Dr. Muhammad Tahir Aziz, Chief Operating Officer, SKMCH\&RC, Peshawar and head of pharmacy Dr. Abdul Wahab from both settings approved the research. Patient consent was taken in Urdu/English language before collecting the data. There were several reasons for verbal consent.

1. The patients in Khyber Pakhtunkhwa are mostly illiterate because of which the researcher need to translate verbally the purpose of study or data collection.

2. No interventions/changes were directly made to any medication order of the patient by the researches.

3. The ethics committee observed no harm to the patients from the data collection procedure and also keeping in mind the patients educational background allowed to take verbal consents.

4. The consent was documented on a consent form designed both in English and Urdu languages.

\section{Consent for publication}

Not applicable.

Competing interests

The authors declare that they have no competing interests.

\section{Author details}

'Quaid-I-Azam University, QAU Islamabad, Islamabad, Pakistan. ${ }^{2}$ Department of Pharmacy, Qauid-I-Azam University, QAU Islamabad, Islamabad, Pakistan. ${ }^{3}$ Institute of Pharmaceutical Science, UVAS Lahore, Lahore, Pakistan. ${ }^{4}$ Riphah International University Islamabad, Islamabad, Pakistan.

Received: 4 February 2019 Accepted: 9 August 2019

Published online: 19 August 2019

\section{References}

1. Otero P, Leyton A, Mariani G, Cernadas JMC. Medication errors in pediatric inpatients: prevalence and results of a prevention program. Pediatrics. 2008; 122(3):e737-e43.

2. Schwappach D, Wernli M. Medication errors in chemotherapy: incidence, types and involvement of patients in prevention. A review of the literature. Eur J Cancer Care (Engl). 2010;19(3):285-92.

3. Samp JC, Touchette DR, Marinac JS, Kuo GM, Collaborative ACoCPPBRN. Economic evaluation of the impact of medication errors reported by US clinical pharmacists. Pharmacotherapy. 2014;34(4):350-7.

4. Mansouri A, Ahmadvand A, Hadjibabaie M, Kargar M, Javadi M, Gholami K. Types and severity of medication errors in Iran; a review of the current literature. DARU Jo Pharma Sci. 2013;21(1):49.

5. Ashcroft DM, Lewis PJ, Tully MP, Farragher TM, Taylor D, Wass V, et al. Prevalence, nature, severity and risk factors for prescribing errors in hospital inpatients: prospective study in 20 UK hospitals. Drug Saf. 2015;38(9):833-43.

6. Aljadhey H, Mahmoud MA, Mayet A, Alshaikh M, Ahmed Y, Murray MD, et al. Incidence of adverse drug events in an academic hospital: a prospective cohort study. Int J Qual Health Care. 2013;25(6):648-55.

7. Kaushal R, Bates DW, Landrigan C, McKenna KJ, Clapp MD, Federico F, et al. Medication errors and adverse drug events in pediatric inpatients. Jama. 2001;285(16):2114-20.

8. O'Shea E. Factors contributing to medication errors: a literature review. J Clin Nurs. 1999:8(5):496-504

9. Wong IC, Ghaleb MA, Franklin BD, Barber N. Incidence and nature of dosing errors in paediatric medications. Drug Saf. 2004;27(9):661-70.

10. Carayon P, Wetterneck TB, Cartmill R, Blosky MA, Brown R, Kim R, et al. Characterising the complexity of medication safety using a human factors approach: an observational study in two intensive care units. BMJ Qual Saf. 2014;23(1):56-65.

11. Mathaiyan J, Jain T, Dubashi B, Reddy KS, Batmanabane G. Prescription errors in cancer chemotherapy: omissions supersede potentially harmful errors. J Pharmacol Pharmacother. 2015:6(2):83-7.

12. Ranchon F, Moch C, You B, Salles G, Schwiertz V, Vantard N, et al. Predictors of prescription errors involving anticancer chemotherapy agents. Eur J Cancer. 2012;48(8):1192-9.

13. Ranchon F, Salles G, Späth H-M, Schwiertz V, Vantard N, Parat S, et al. Chemotherapeutic errors in hospitalised cancer patients: attributable damage and extra costs. BMC Cancer. 2011;11:478.

14. Walsh KE, Dodd KS, Seetharaman K, Roblin DW, Herrinton LU, Von Worley A, et al. Medication errors among adults and children with cancer in the outpatient setting. J Clin Oncol. 2008;27(6):891-6.

15. Watts RG, Parsons K. Chemotherapy medication errors in a pediatric cancer treatment center: prospective characterization of error types and frequency and development of a quality improvement initiative to lower the error rate. Pediatr Blood Cancer. 2013;60(8):1320-4

16. FitzGerald RJ. Medication errors: the importance of an accurate drug history Br J Clin Pharmacol. 2009;67(6):671-5.

17. Tang Fl, Sheu SJ, Yu S, Wei IL, Chen CH. Nurses relate the contributing factors involved in medication errors. J Clin Nurs. 2007:16(3):447-57.

\section{Publisher's Note}

Springer Nature remains neutral with regard to jurisdictional claims in published maps and institutional affiliations. 